\title{
An Appraisal of Power-Minimizing Control Algorithms for Active Magnetic Bearings
}

\author{
Seong-yeol Yoo' and Myounggyu D. Noh ${ }^{2}$ \\ ${ }^{1}$ Ocean System Engineering Research Division, Korea Research Institute of Ships \& Ocean Engineering, \\ Daejeon 305-343, Republic of Korea \\ ${ }^{2}$ Department of Mechatronics Engineering, Chungnam National University, Daejeon 305-764, Republic of Korea \\ Correspondence should be addressed to Myounggyu D. Noh; mnoh@cnu.ac.kr
}

Received 23 October 2014; Revised 18 January 2015; Accepted 20 January 2015

Academic Editor: Mohammad Elahinia

Copyright (c) 2015 S.-y. Yoo and M. D. Noh. This is an open access article distributed under the Creative Commons Attribution License, which permits unrestricted use, distribution, and reproduction in any medium, provided the original work is properly cited.

Active magnetic bearings consume much less power than conventional passive bearings, especially when power-minimizing controllers are employed. Several power-minimizing controllers have been proposed, such as variable bias controllers and switching controllers. In this paper, we present an appraisal of the power-minimizing control algorithms for active magnetic bearings in an attempt to provide an objective guideline on the merits of the control algorithms. In order for the appraisal, we develop an unified and consistent model of active magnetic bearing systems. The performances of the power-minimizing controllers are assessed through this model. The results show that the power-minimizing controllers indeed save considerable power when the machine state is relatively steady. However, a simple proportional-derivative type controller is on a par with the much more complex powerminimizing controllers in terms of power consumption when the machine is experiencing transient loads.

\section{Introduction}

Magnetic bearings suspend a rotor without contact using magnetic forces. Compared to more conventional bearings such as rolling-element and fluid bearings, magnetic bearings enable higher speeds and lower losses and operations in cryogenic temperatures, vacuum, or other extreme environments. Magnetic bearings are classified into two categories: passive and active. Passive magnetic bearings use permanent magnets or superconductors and do not require feedback control. Active magnetic bearings (AMBs) use electromagnets for generating magnetic forces and are open-loop unstable. Thus, a feedback control is necessary. Typically, AMBs offer much greater capability in vibration control than the passive counterparts. AMBs have been applied to numerous machines such as compressors, pumps, and turboexpanders. A recent survey of AMB applications can be found in [1].

The force-current relationship in an AMB is quadratic. In order to use linear control laws and also to guarantee finite slew rate in force, the bias current linearization is typically employed. The coil currents consist of constant bias currents and dynamic control currents. The bias currents maintain static equilibrium, while the control currents react to the disturbances. A downside of the bias linearization is that electrical power must be consumed to maintain constant bias currents.

In order to reduce the power consumption of AMBs due to bias currents, several power-minimizing control algorithms have been proposed. These power-minimizing algorithms either eliminate bias currents (zero bias) [2-4] or use low-bias currents [5-8]. All zero-bias methods involve a certain kind of switching and are affected by the singularity at the zero-bias state. Low-bias algorithms adjust bias currents according to adaptive or predetermined rules.

Although they would definitely reduce the power consumption when compared to a controller with fixed bias currents, no research has been done to objectively compare the power-minimizing control laws. Authors of the existing work on power-minimizing controllers compare the power-savings of their controller with a reference controller, 
typically a proportional-integral-derivative (PID) type. No attempts have been made to compare the performance of a power-minimizing controller with another power-saving algorithm. When selecting a power-minimizing controller, it is crucial to assess when and how much the controller can save power consumption in comparison with a reference controller or other power-minimizing control algorithms. Furthermore, the controllers to be compared must have the same characteristics in some senses.

For a fair comparison of the controllers, it is critical to have a common platform that the performance of the controller is assessed. An experimental test-rig would not serve this purpose for two reasons. First, it is almost impossible to exclude side factors that are related to a test-rig. Thus, it would be difficult to judge whether the difference in powersaving is due to the control algorithms or other side factors such as switching noise in power amplifiers, the level of which usually changes with respect to the magnitude of currents. Second, a test-rig is not adequate to simulate the situation when the controller fails to operate as required. For example, a switching controller may fail when there is a significant phase delay between the magnetic force and the resulting displacement of the suspending object. For these reasons, a virtual test-rig would be ideal to give us at least a guideline to the efficacy of the power-minimizing control algorithms.

Assessing and predicting the efficacy of a control algorithm become straightforward if the system that combines the plant and the controller is linear. An especially convenient tool for a linear system is a frequency-domain technique using the maximum singular values (SV) that provide essential information about the system without intensive timedomain simulations. A speedy check on the controller performance makes it possible to run an extensive parametric search in order to find an optimal set of controller gains.

In this paper, we developed a unified and consistent model for AMB systems as a virtual test-rig. The performance of the power-minimizing controllers is assessed through this model using frequency-domain techniques. Of many power-minimizing controllers in the literature, we select two controllers for comparison: the variable bias controller by Sahinkaya and Hartavi [8] and the switching controller by Sivrioglu et al. [4], partly because these controllers were experimentally validated. A more important reason for this selection is that although these two controllers are either nonlinear or adaptive, it is still possible to use the SV-based frequency-domain technique for the assessment. Other nonlinear control algorithms such as the backstepping controller [5] or the passivity-based controller [6] do not permit the use of linear techniques and thus are excluded from the comparisons.

A reference controller is designed, against which the power-minimizing controllers are compared. The reference controller is a proportional-derivative (PD) type controller. A synchronous notch filter can be applied if the disturbance is mostly due to unbalance in rotor. The controllers were designed to give the same closed-loop stiffness at low rotational speeds in order to ensure an objective comparison.

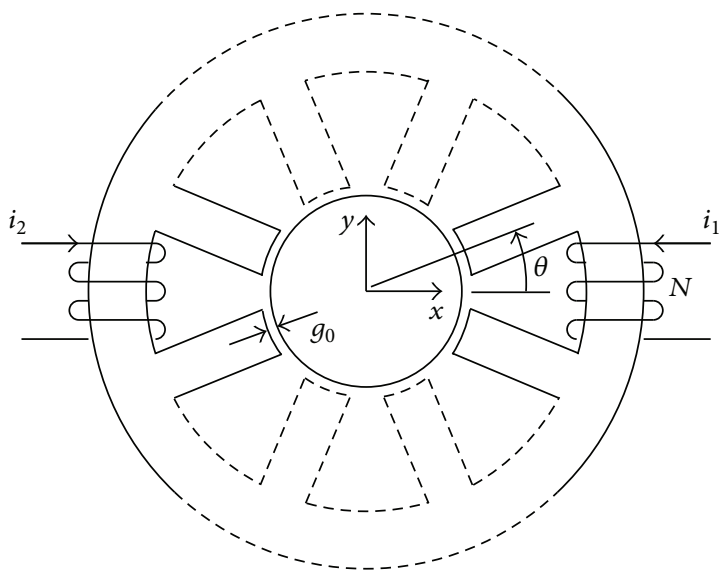

FIGURE 1: Eight-pole radial magnetic bearing.

\section{Bias Linearization for AMB}

Magnetic bearings suspend the rotor using the force generated from the magnetic field in the air gap between the bearing and the rotor. The generated force is nonlinear with respect to the coil currents and the air gap lengths. Referring to the schematic of a radial magnetic bearing displayed in Figure 1, the force in the horizontal direction can be written as [1]

$$
f_{\text {mag }}=c_{f}\left[\frac{i_{1}^{2}}{\left(g_{0}-x \cos \theta\right)^{2}}-\frac{i_{2}^{2}}{\left(g_{0}+x \cos \theta\right)^{2}}\right] .
$$

The force factor, $c_{f}$, in (1) is defined as

$$
c_{f}=\frac{\mu_{0} N^{2} A_{g} \cos \theta}{4},
$$

where $\mu_{0}$ is the permeability of free space $\left(=4 \pi \times 10^{-7} \mathrm{H} / \mathrm{m}\right)$, $A_{g}$ the pole face area, $N$ the number of coil turns, and $\theta$ the pole angle.

In order to be able to apply a linear controller, the force model of (1) can be linearized in various ways $[9,10]$. The most common method of linearization is often referred to as bias linearization, where the opposing coil currents are diametrically perturbed by control currents from the constant bias current, which can be written as

$$
\begin{aligned}
& i_{1}=\max \left(0, I_{b}+i_{p}\right), \\
& i_{2}=\max \left(0, I_{b}-i_{p}\right) .
\end{aligned}
$$

Since the force is only proportional to the square of coil currents, the coil currents are not allowed to be negative. A condition to obtain a linear force-current relationship is

$$
\left|i_{p}\right|<I_{b}
$$

A popular choice of the bias current for class-A operation is

$$
I_{b}=0.5 I_{\max }
$$




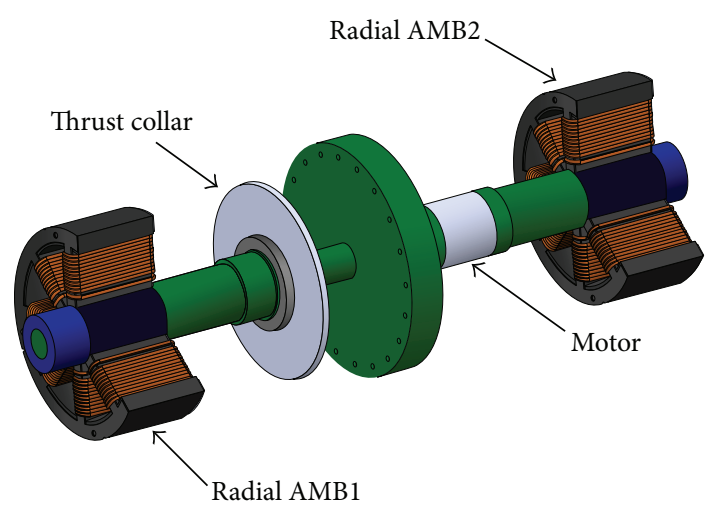

FIgURE 2: The rotor and radial AMBs in the controller test bed.

where $I_{\max }$ is the maximum current that can be supplied from the amplifier [11].

Using the current law of (3) and performing Taylor series expansion on the force model of (1), we get a linearized force law:

$$
f \approx-K_{x} x+K_{i} i_{p}
$$

where the actuator gain, $K_{i}$, and the actuator open-loop stiffness, $K_{x}$, are defined as

$$
\begin{aligned}
K_{i} & =4 c_{f} \frac{I_{b}}{g_{0}^{2}}, \\
K_{x} & =-K_{i} \frac{I_{b}}{g_{0}} \cos \theta .
\end{aligned}
$$

Since the open-loop stiffness is negative, the linearized magnetic bearing is unstable, and it must be stabilized through feedback control.

Due to coil resistances, the currents in the coils produce power losses. If the control current is sinusoidal, the rootmean-square (RMS) power loss due to the current in one coil is

$$
P_{\mathrm{RMS}}=\frac{R_{\mathrm{coil}}}{2 \pi} \int_{0}^{2 \pi}\left(I_{b}+i_{p} \sin \phi\right)^{2} d \phi=\left(2 I_{b}^{2}+i_{p}^{2}\right) R_{\mathrm{coil}} .
$$

Thus, even if the magnitude of the control current is the same as the bias current, the power loss due to the bias current is twice that due to the control current. This is why most of the power-minimizing control algorithms try to minimize (or eliminate) the bias current.

In order to appraise control algorithms, we designed a rotor system with two sets of radial magnetic bearings and a set of thrust magnetic bearing. The rotor and radial magnetic bearings are illustrated in Figure 2. The specifications of the system are summarized in Table 1.

\section{Simulation Model}

An AMB system consists of a rotor, displacement sensors, amplifiers, and controllers. When comparing different control algorithms, it is of utmost important to maintain the
TABLE 1: Specifications of the controller test bed.

\begin{tabular}{cccc}
\hline & Parameter & Value & Unit \\
\hline \multirow{4}{*}{ Rotor } & Mass & 16.6 & $\mathrm{~kg}$ \\
& Length & 0.624 & $\mathrm{~m}$ \\
& Polar moment of inertia & 0.043 & $\mathrm{~kg}-\mathrm{m}^{2}$ \\
& Transverse moment of inertia & 0.32 & $\mathrm{~kg}-\mathrm{m}^{2}$ \\
& First bending freq. (free-free) & 234 & $\mathrm{~Hz}$ \\
& Second bending freq. (free-free) & 600 & $\mathrm{~Hz}$ \\
& Maximum speed & 18,000 & $\mathrm{rpm}$ \\
\hline \multirow{4}{*}{ AMB 1,2 } & Pole face area, $A_{g}$ & 900 & $\mathrm{~mm}^{2}$ \\
& Nominal air gap, $g_{0}$ & 0.6 & $\mathrm{~mm}$ \\
& Backup bearing clearance & 0.3 & $\mathrm{~mm}$ \\
& Pole angle, $\theta$ & 22.5 & $\mathrm{deg}$. \\
& Coil turns, $N$ & 100 & - \\
\hline
\end{tabular}

plant that each controller sees as identical as possible. In this sense, a simulation study is ideal for this purpose as long as the model describes the system accurately. In this paper, we develop a state-space model of the system that takes such factors into account as flexibility of the rotor, bandwidths of amplifiers, antialiasing filters, and sampling delay. The model is also dependent on the running speed of the rotor, mainly because of the gyroscopic coupling. Therefore, time-domain simulations would take tremendous computational time for parametric studies. In contrast, simulations with parameter variations can be conveniently done in frequency-domain. Furthermore, the use of singular values for rotordynamic analyses [12] facilitates the controller comparison.

3.1. Rotor Model. The rotor is the most important element in an AMB system in a sense that the system dynamics is largely determined by the dynamics of the rotor. In some cases, a simple rigid-body rotor model is sufficient to describe the system dynamics [13]. In other cases, the rotor model must be detailed enough to capture the dynamics due to the flexibility of the rotor, misalignments, asymmetry, and so forth. In this paper, we first set up a finite-element (FE) model based on slender beam theory [14]. Since a FE model is typically too large, we reduce the model size using modal truncation [15]. The reduced-order model contains two rigidbody modes and two flexible modes. The frequencies of the flexible modes are validated against a full-blown FE model (12475 elements) using ANSYS (ANSYS, Inc., Canonsburg, $\mathrm{PA})$. Figure 3 compares the reduced-order model with the rigid-body and full-order FE model in the form of the transfer function from the force input at the bearing to the sensor output.

3.2. Sensor and Amplifier Model. The displacement sensors in an AMB system measure the rotor displacements and provide feedback signals to the controller. Eddy-current type sensors are commonly used. Although commercial eddycurrent sensors have fairly wide bandwidth, we must consider the dynamics of the sensors due to antialiasing filters and/or low-pass filters in the process of signal conditioning. When 


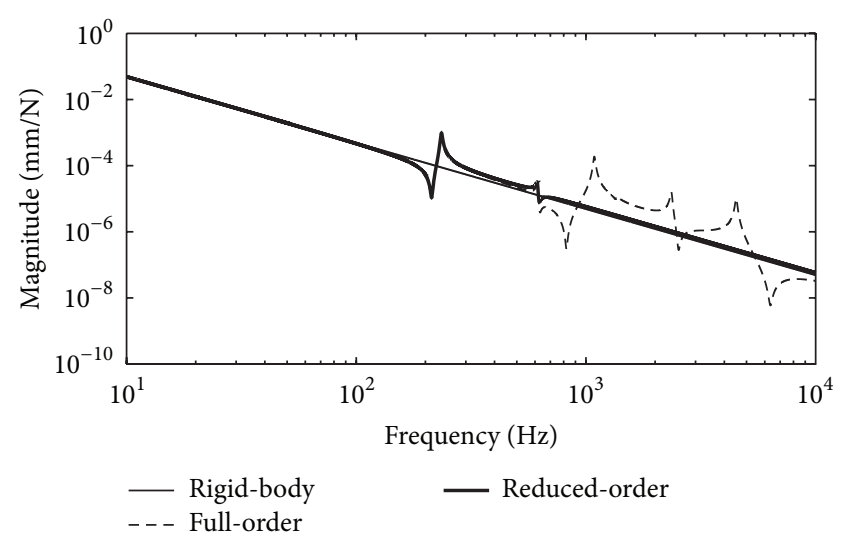

FIgURE 3: Reduced-order rotor model in frequency domain. Rigidbody model and full FE model are given for comparison.

a digital controller is used, the analog sensor signals must be converted into digital forms. Digital systems have inherently sampling delays. The model for sensors must include the effect of sampling delays, which can be approximated as a filter using Pade approximation [1].

In this paper, it is assumed that the sensors are of a commercially available eddy-current type (PU5505, AEC, Japan) which has the bandwidth of up to $24 \mathrm{kHz}$. The antialiasing filter is a second-order low-pass filter with the cut-off frequency of $1 \mathrm{kHz}$. The sampling delay of the digital controller system that we have is measured to be $200 \mu$ s at the sampling rate of $10 \mathrm{kHz}$.

As for the amplifier, we measure the frequency response of a commercial transconductance amplifier (JSP-180-20, Copley Controls) with a load having similar impedance as the bearing coils. Using a curve-fitting technique, a second-order low-pass type model is identified as a model for the amplifier. The cut-off frequency and the damping ratio are estimated to be $1 \mathrm{kHz}$ and 0.7 , respectively.

3.3. System Model. The aforementioned models for the rotor, sensors, and amplifiers can be assembled into a plant model following the synthesis technique described in [1]. The resulting plant model can be expressed in a state-space form as

$$
\begin{aligned}
\frac{d}{d t} \mathbf{x}_{\text {plant }} & =\mathbf{A}_{\text {plant }} \mathbf{x}_{\text {plant }}+\mathbf{B}_{\text {plant }, u} \mathbf{u}+\mathbf{B}_{\text {plant }, w} \mathbf{w}, \\
\mathbf{z} & =\mathbf{C}_{\text {plant }, z} \mathbf{x}_{\text {plant }}+\mathbf{D}_{\text {plant }, u z} \mathbf{u}, \\
\mathbf{y} & =\mathbf{C}_{\text {plant }, y} \mathbf{x}_{\text {plant }}+\mathbf{D}_{\text {plant }, w y} \mathbf{w} .
\end{aligned}
$$

The size of this model is rather huge. For example, the model we use in this paper has the state matrix $\mathbf{A}_{\text {plant }}$ of $48 \times 48$. The specifics of the model can be found in [1].

In (9), two input signals, $\mathbf{u}$ and $\mathbf{w}$, denote the controller output and the disturbance to the plant, respectively. Rotor unbalance and sensor noises are the disturbances that are considered in this paper. Signal $\mathbf{z}$ contains the displacements at the user-defined points along the rotor, coil currents, applied voltages, and flux densities, which are utilized for

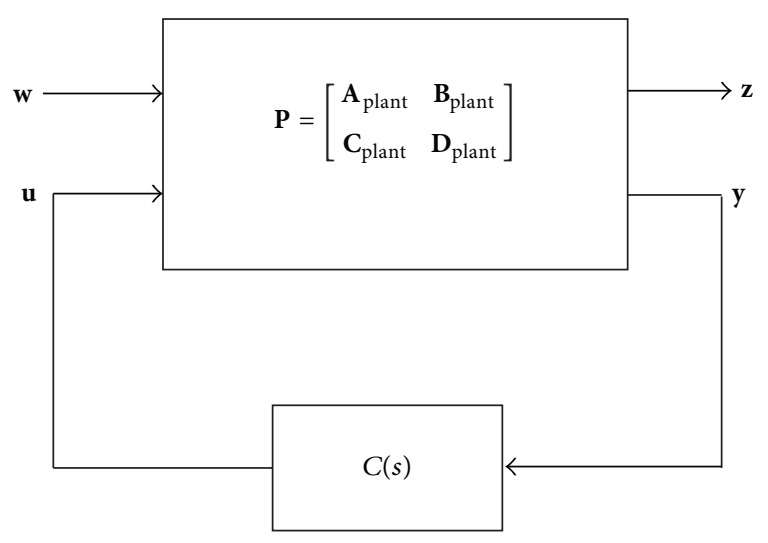

FIGURE 4: System block diagram.

performance measure. Signal y consists of the sensor outputs. Matrix $\mathbf{A}_{\text {plant }}$ is a function of the running speed because of the gyroscopic coupling. It is assumed that the axial dynamics is decoupled from the radial dynamics of the rotor, and only the radial dynamics is considered in this paper.

If a linear controller is given, it can be synthesized with the plant model, resulting in a system model:

$$
\begin{aligned}
\frac{d}{d t} \mathbf{x}_{\mathrm{sys}} & =\mathbf{A}_{\mathrm{sys}} \mathbf{x}_{\mathrm{sys}}+\mathbf{B}_{\mathrm{sys}} \mathbf{w}, \\
\mathbf{z} & =\mathbf{C}_{\mathrm{sys}} \mathbf{x}_{\mathrm{sys}} .
\end{aligned}
$$

The block diagram of the system model is illustrated in Figure 4, where $C(s)$ is the transfer function of the controller. If we consider only the unbalance disturbance, signal $\mathbf{w}$ can be written as

$$
\mathbf{w}=m e \Omega^{2}\left[\begin{array}{ll}
1 & 0 \\
0 & 1
\end{array}\right] \overline{\mathbf{w}}=\mathbf{W}_{w} \overline{\mathbf{w}},
$$

where $\Omega$ is the running speed, $m e$ is the unbalance, and $\overline{\mathbf{w}}$ is a unit vector. The closed-loop transfer function from disturbance $\mathbf{w}$ to the performance measure $\mathbf{z}$ is then obtained from

$$
\mathbf{z}=\mathbf{C}_{\text {sys }}\left(s \mathbf{I}-\mathbf{A}_{\text {sys }}\right)^{-1} \mathbf{B}_{\text {sys }} \mathbf{W}_{w} \overline{\mathbf{w}}=\mathbf{G}_{\mathrm{cl}}(s ; \Omega) \mathbf{W}_{w} \overline{\mathbf{w}} .
$$

If the performance measure is normalized by an output weighting matrix $\mathbf{W}_{z}$, then we can write

$$
\overline{\mathbf{z}}=\mathbf{W}_{z} \mathbf{G}_{\mathrm{cl}}(s ; \Omega) \mathbf{W}_{w} \overline{\mathbf{w}} .
$$

Since the system dynamics change with respect to the running speed, we need to inspect the performance measure at a specific speed such that

$$
|\mathbf{z}|=\left|\mathbf{W}_{z} \mathbf{G}_{\mathrm{cl}}(s ; \Omega) \mathbf{W}_{w}\right|_{s=j \Omega} .
$$

The singular value decomposition is a useful tool for rotordynamic analyses, since it can tell us the worst case [12]. If the signals are normalized as in (13), the condition that

$$
\bar{\sigma}\left(\mathbf{W}_{z} \mathbf{G}_{\mathrm{cl}}(s ; \Omega) \mathbf{W}_{w}\right)_{s=j \Omega}<1
$$

is equivalent to that any of the outputs are below the performance limit due to any combination of the inputs. 


\section{Control Algorithms}

Since an active magnetic bearing is open-loop unstable, a feedback control is necessary. The controller for AMB systems must not only satisfy performance requirements, but also maintain system stability. When designing a controller for AMB system, a care must be taken because the dynamics of a rotating system can change with respect to the running speed. Furthermore, the presence of a flexible mode in the operating speed range makes the controller design more involved.

In this paper, we assume that the disturbance to the system is mostly rotor unbalance. The performance requirements are as follows.

(i) The excursions at the bearings must stay in the clearance.

(ii) The coil current must be smaller than the maximum current that the amplifier can provide.

A reference controller is first designed that satisfies these performance requirements as well as the stability condition in terms of the output sensitivity. Then, two power-minimizing controllers are designed in such a way that the unbalance responses follow the case with the reference controller as closely as possible.

4.1. Reference Controller. A common type of the controller for $\mathrm{AMB}$ is a proportional-derivative (PD) controller, where the proportional gain, $K_{P}$, determines the closed stiffness of the bearing, and the derivative gain, $K_{D}$, is related to damping. The PD controller is alternatively called a phase lead filter, because the filter provides positive phase shift in the frequency region where maximum damping is necessary. The PD controller that we adopted in this paper is in the form of

$$
C(s)=K_{P} \frac{\omega_{\text {con }}^{2}\left(\tau_{D} s+1\right)}{s^{2}+2 \zeta_{\text {con }} \omega_{\text {con }} s+\omega_{\text {con }}^{2}},
$$

where $\tau_{D}=K_{D} / K_{P}$. A second-order filter is appended to the ideal PD controller of $K_{P}\left(\tau_{D} s+1\right)$ to limit the gain at high frequencies.

The presence of the flexible mode in the operating range increases the output sensitivity or destabilizes the system at worst. In order to operate beyond the flexible mode, a notch filter $[16,17]$ can be used. In this paper, we have used a notch filter in the form of

$$
N(s)=\frac{\tau_{\mathrm{nz}}^{2} s^{2}+2 \zeta_{\mathrm{nz}} \tau_{\mathrm{nz}} s+1}{\tau_{\mathrm{np}}^{2} s^{2}+2 \zeta_{\mathrm{np}} \tau_{\mathrm{np}} s+1} .
$$

The controller transfer function is then

$$
C(s)=K_{P} \frac{\omega_{\text {con }}^{2}\left(\tau_{D} s+1\right)}{s^{2}+2 \zeta_{\mathrm{con}} \omega_{\mathrm{con}} s+\omega_{\mathrm{con}}^{2}} \cdot N(s) .
$$

When the unbalance is the dominant disturbance, a synchronous notch filter [18] can reduce the response and subsequently power consumptions. The controller of (18) is appended by a synchronous notch filter given as

$$
N_{\text {sync }}(s ; \Omega)=\frac{s^{2}+2 \zeta_{\text {sync1 }} \Omega+\Omega^{2}}{s^{2}+2 \zeta_{\text {sync } 2} \Omega+\Omega^{2}} .
$$
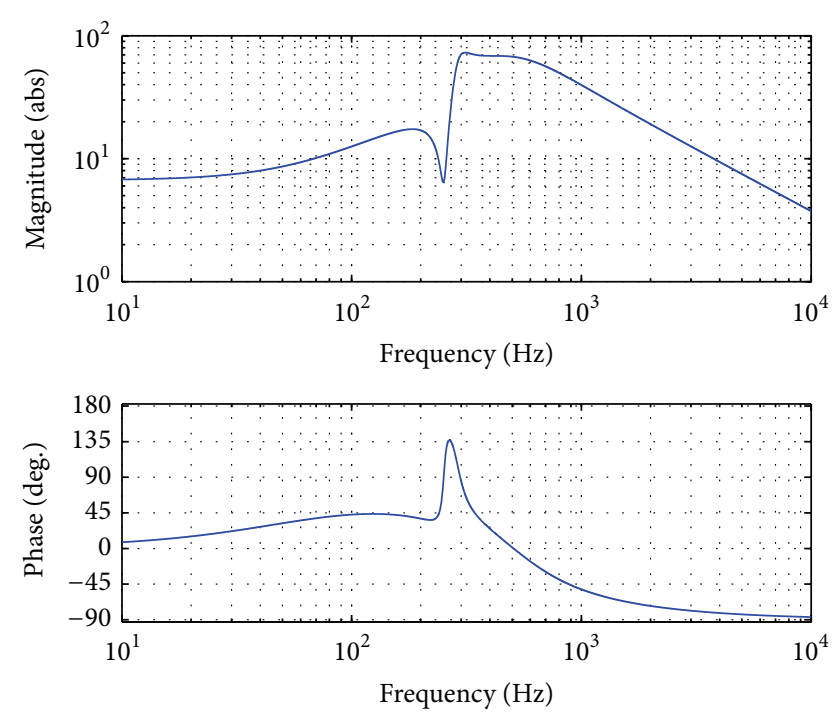

FIGURE 5: Reference controller.

TABLE 2: Parameters for the reference PD controller.

\begin{tabular}{lccc}
\hline Parameter & Symbol & Value & Unit \\
\hline Proportional gain & $K_{P}$ & 9.535 & $\mathrm{~V} / \mathrm{mm}$ \\
Derivative gain & $K_{D}$ & 0.0253 & $\mathrm{~V} \cdot \mathrm{s} / \mathrm{mm}$ \\
Controller pole & $\omega_{\text {con }}$ & 3204 & $\mathrm{rad} / \mathrm{s}$ \\
Controller damping & $\zeta_{\text {con }}$ & 0.6 & - \\
Time constant for notch zero & $\tau_{\mathrm{nz}}$ & 0.555 & $\mathrm{~ms}$ \\
Damping for notch zero & $\zeta_{\mathrm{nz}}$ & 0.03 & - \\
Time constant for notch pole & $\tau_{\mathrm{np}}$ & 0.631 & $\mathrm{~ms}$ \\
Damping for notch pole & $\zeta_{\mathrm{np}}$ & 0.09 & - \\
Damping for synchronous notch zero & $\zeta_{\text {sync1 }}$ & 0.06 & - \\
Damping for synchronous notch pole & $\zeta_{\text {sync2 }}$ & 0.08 & - \\
\hline
\end{tabular}

The final form of the reference controller is thus given by

$$
C(s)=K_{P} \frac{\omega_{\text {con }}^{2}\left(\tau_{D} s+1\right)}{s^{2}+2 \zeta_{\text {con }} \omega_{\text {con }} s+\omega_{\text {con }}^{2}} \cdot N \cdot N_{\text {sync }} .
$$

The parameters of the reference controller are summarized in Table 2. The bode plot of the controller is shown in Figure 5. For the reference controller, the bias current of $2 \mathrm{~A}$ is used, resulting in the actuator gain of $58.05 \mathrm{~N} / \mathrm{A}$ and the openloop stiffness of $179.8 \mathrm{~N} / \mathrm{mm}$. The maximum current that the amplifier can provide is $4 \mathrm{~A}$.

The reference controller provides plenty of stability margin. According to ISO standard [19], it is desirable for a machine equipped with active magnetic bearings to have the output sensitivity below $9.5 \mathrm{~dB}$ (zone A). Figure 6 shows the output sensitivity of the closed-loop system with the reference controller. The sensitivity is mostly in zone A with a brief exception around $2200 \mathrm{rpm}$.

4.2. Variable Bias Controller. The variable bias controller [8] adjusts the bias currents according to the synchronous 


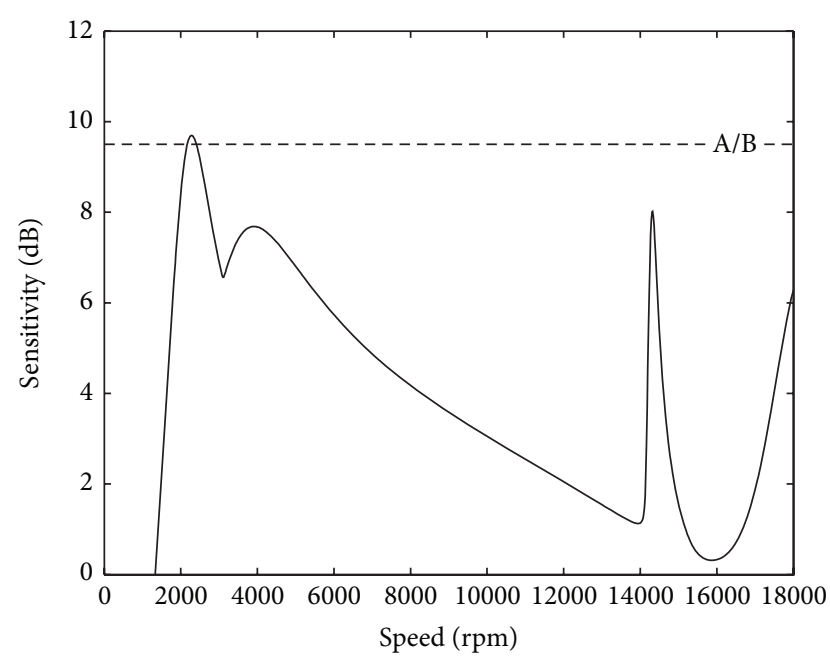

FIGURE 6: Output sensitivity of the reference controller.

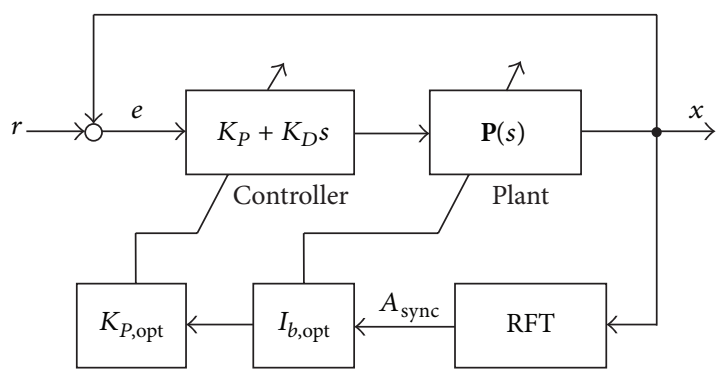

FIgURE 7: Variable bias controller.

response and the desired closed-loop stiffness. As long as the class-A operation is valid, the optimal bias is

$$
I_{b, \mathrm{opt}}=\frac{1}{4} \sqrt{\frac{g_{0}^{3} A_{\mathrm{sync}} K_{\mathrm{cl}}}{c_{f}\left(g_{0}-A_{\mathrm{sync}} \cos \theta\right)}},
$$

where $A_{\text {sync }}$ is the synchronous response and $K_{\mathrm{cl}}$ is the desired closed-loop stiffness. The upper limit of the optimal bias is the bias for the reference controller. Once the bias current is determined, the proportional gain is adjusted to achieve $K_{\mathrm{cl}}$ according to

$$
K_{P, \mathrm{opt}}=\frac{I_{b, \mathrm{opt}}}{g_{0}}+\frac{g_{0}^{2} K_{\mathrm{cl}}}{4 c_{f} I_{b, \mathrm{opt}}} .
$$

Figure 7 illustrates the structure of the variable bias controller. The recursive Fourier transform (RFT) extracts the synchronous component from the sensor measurements. The optimal bias current and the proportional gain are computed by (21) and (22), respectively.

4.3. Switching Controller. Using the feedback linearization, the switching controller by Sivrioglu et al. [4] linearizes the plant through a switching rule. Referring to the $1 \mathrm{D}$ actuator in Figure 1, a dynamic model for the rotor can be written as

$$
m \ddot{x}=f_{\text {mag }} .
$$

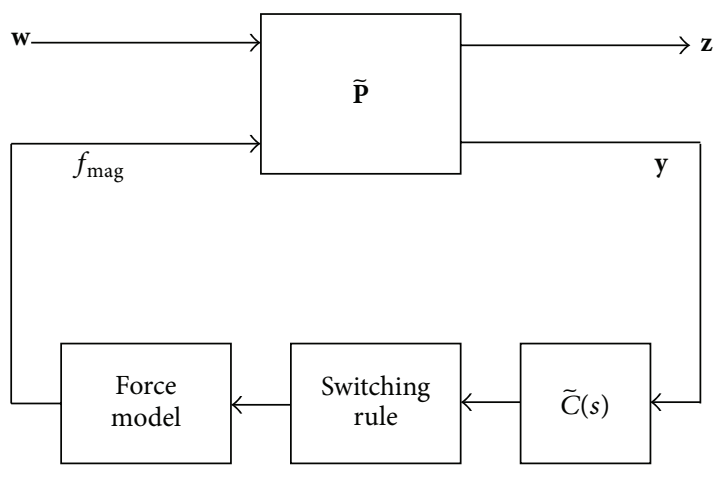

Figure 8: Switching controller.

In other words, the plant model is linear with respect to the force. If the coil currents are determined by

$$
\begin{aligned}
& i_{1}=0, \quad i_{2}=\left(g_{0}+x\right) \sqrt{\frac{\left|f_{\mathrm{mag}}\right|}{c_{f}}}, \quad \text { if } x>0, \\
& i_{1}=\left(g_{0}-x\right) \sqrt{\frac{\left|f_{\mathrm{mag}}\right|}{c_{f}}}, \quad i_{2}=0, \quad \text { if } x<0,
\end{aligned}
$$

the plant seen by the controller is linear with $f_{\text {mag }}$ as the control input. In order for an objective comparison with other control algorithms, the proportional gain of the reference controller is adjusted so that the force acting on the rotor is identical at least in low speed regions. The plant model must be modified accordingly. The structure of the switching controller is illustrated in Figure 8.

Because of the switching rule of (24), the RMS power calculation by (8) must be modified for the switching control algorithm. Assuming that the displacement and the force are purely sinusoidal, expressed as

$$
\begin{gathered}
x=X \sin \omega t, \\
f_{\text {mag }}=-F_{\text {mag }} \sin \omega t ;
\end{gathered}
$$

the squares of the coil currents are

$$
\begin{aligned}
& i_{1}^{2}=0, \\
& i_{2}^{2}=\left(g_{0}+X \sin \omega t\right)^{2}\left(\frac{F_{\text {mag }} \sin \omega t}{c_{f}}\right)
\end{aligned}
$$

during $0<t<\pi / \omega$ and

$$
\begin{aligned}
& i_{1}^{2}=\left(g_{0}-X \sin \omega t\right)^{2}\left(\frac{F_{\mathrm{mag}} \sin \omega t}{c_{f}}\right), \\
& i_{2}^{2}=0
\end{aligned}
$$

during $\pi / \omega<t<2 \pi / \omega$. The RMS ohmic loss is

$$
P_{\mathrm{RMS}}=\frac{\omega}{2 \pi} \int_{0}^{2 \pi / \omega}\left(R_{\mathrm{coil}} i_{1}^{2}+i_{2}^{2}\right) d t .
$$




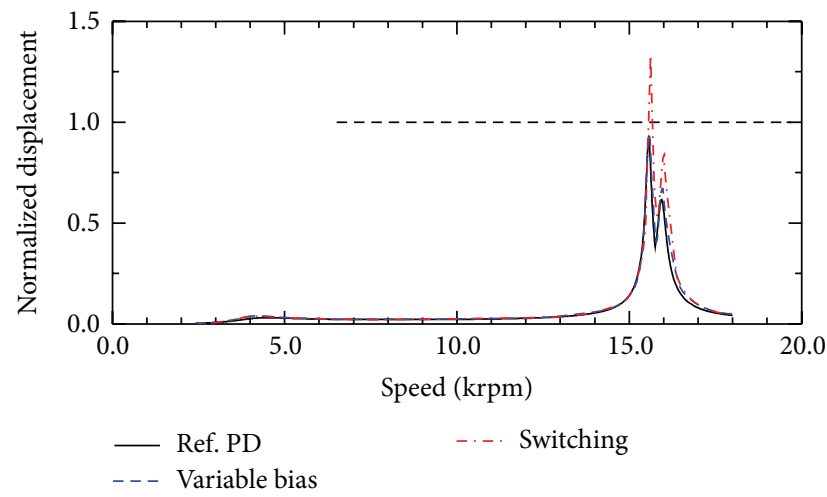

(a)

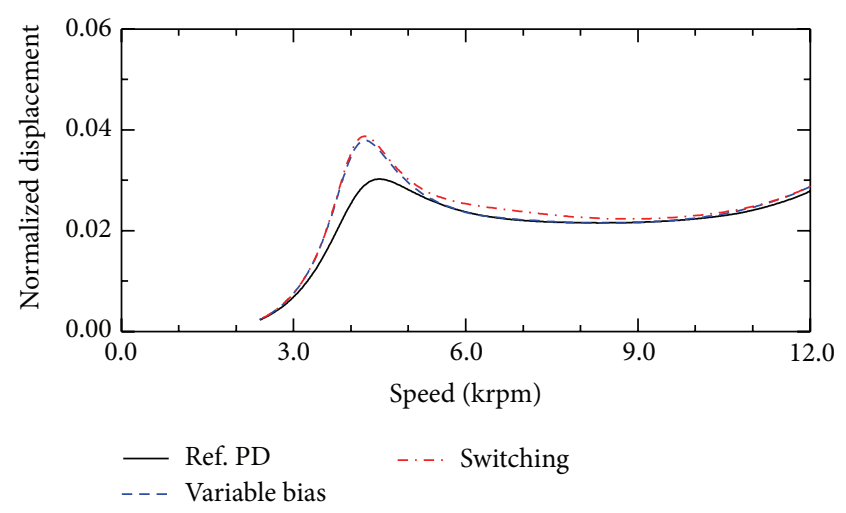

(b)

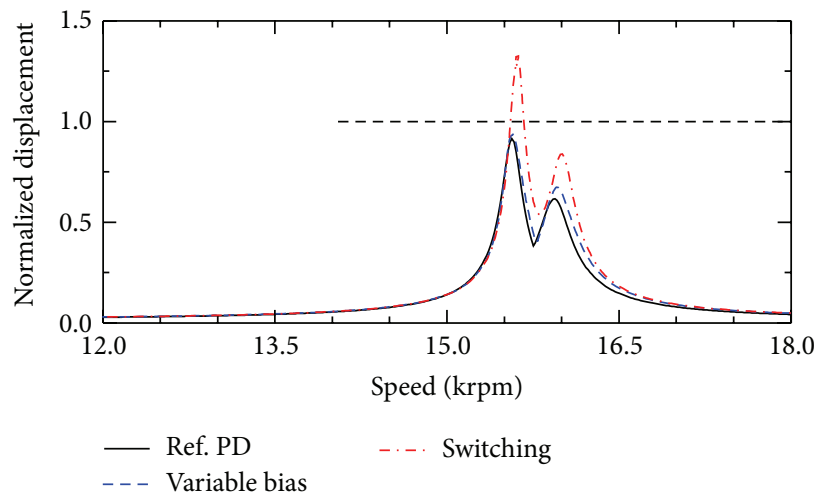

(c)

Figure 9: Unbalance response (displacement).

Substituting (26) and (27) into (28) and performing integration, we get

$$
P_{\mathrm{RMS}}=\frac{R_{\mathrm{coil}} F_{\mathrm{mag}}}{\pi c_{f}}\left(2 g_{0}^{2}+\pi g_{0} X+\frac{4}{3} X^{2}\right) .
$$

\section{Results and Discussion}

Maximum singular values are a convenient tool to assess the possibility of excessive vibrations in rotordynamic analyses [12]. If the input and output are normalized with respect to their bounds, we only need to check the condition of (15). If (15) is satisfied, there is no possibility that the response can be higher than the bounds. Figure 9(a) is the normalized maximum singular values of the radial displacements at the bearings, when the rotor is spinning up from zero to $18,000 \mathrm{rpm}$. Figures 9(b) and 9(c) are the enlarged versions of Figure 9(a) in low and high speed ranges, respectively, which show the similarities and disparities of the control algorithms.

Referring to Figure 9(b), three control laws produce almost the same responses at low speeds except at around $4200 \mathrm{rpm}$ which is the rigid-body mode. The fact that the responses are similar indicates that their closed-loop dynamics are comparable in terms of the closed-loop stiffness. The discrepancy around the rigid-body mode also illustrates when the control laws differ, the point which is evident at the flexible mode.

As can be seen in Figure 9(c), the responses of the systems employing three control laws are very different, when the rotor is passing through critical speeds related to the flexible mode of the rotor (around 15,500 rpm). For the switching control law, the response is greater than the bearing clearance, which means that the system is inoperable and is not able to go above this speed. It is very likely that the switching control law is unable to provide enough stability margin. In contrast, the other two controllers keep the response within the bearing clearance.

The coil currents are an indication of control efforts and also directly related to the ohmic losses. Figure 10 displays the maximum total current in the bearing coils obtained while the rotor is subject to the unbalance disturbance from zero to $18,000 \mathrm{rpm}$. As is clearly shown in Figure 10(b), the peaks of the maximum currents are almost equal, occurring when the rotor passes the flexible mode irrespective of the control algorithms. This means that the coil currents are not a suitable indicator for determining whether the controllers are working properly or not when the system is operating near critical speeds. It also means that the results above the critical speed are not trustworthy for the switching controller case. However, the results below this critical speed are useful. As expected, the variable bias and switching control laws require 


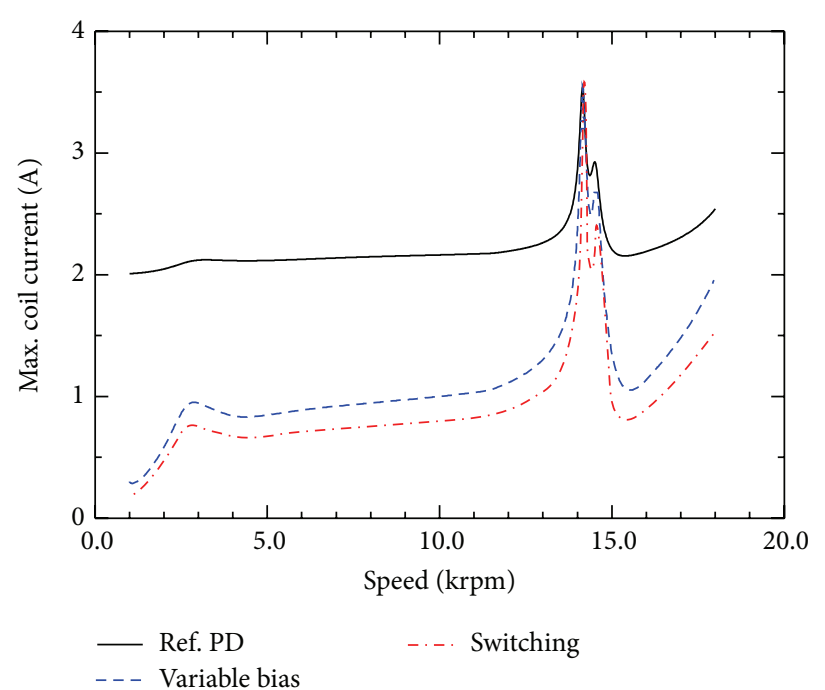

(a)

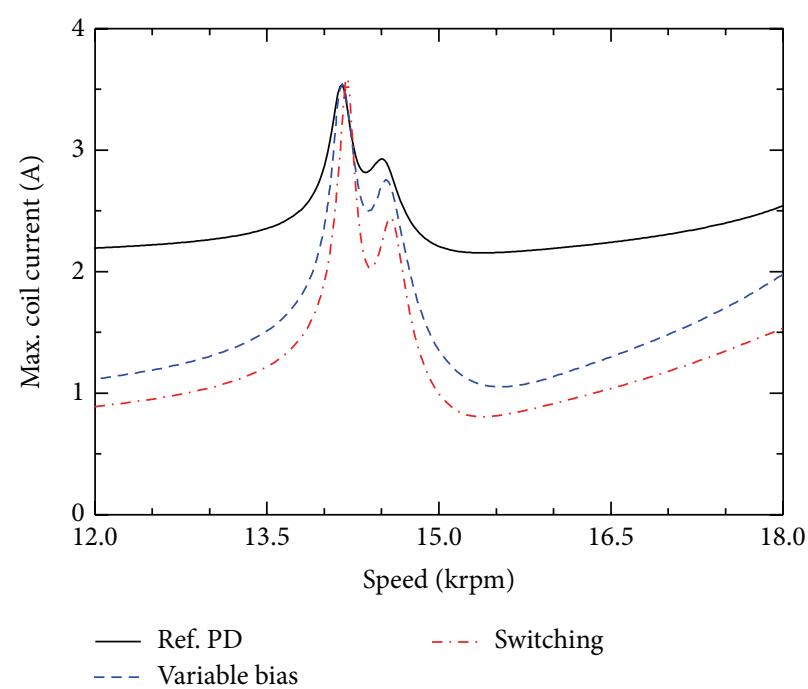

(b)

FIGURE 10: Unbalance response (displacement).

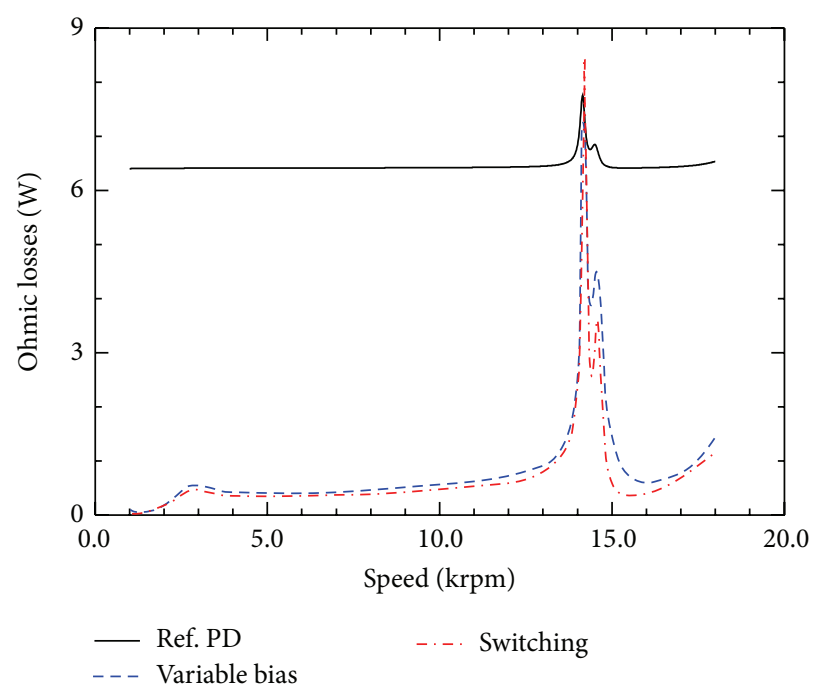

(a)

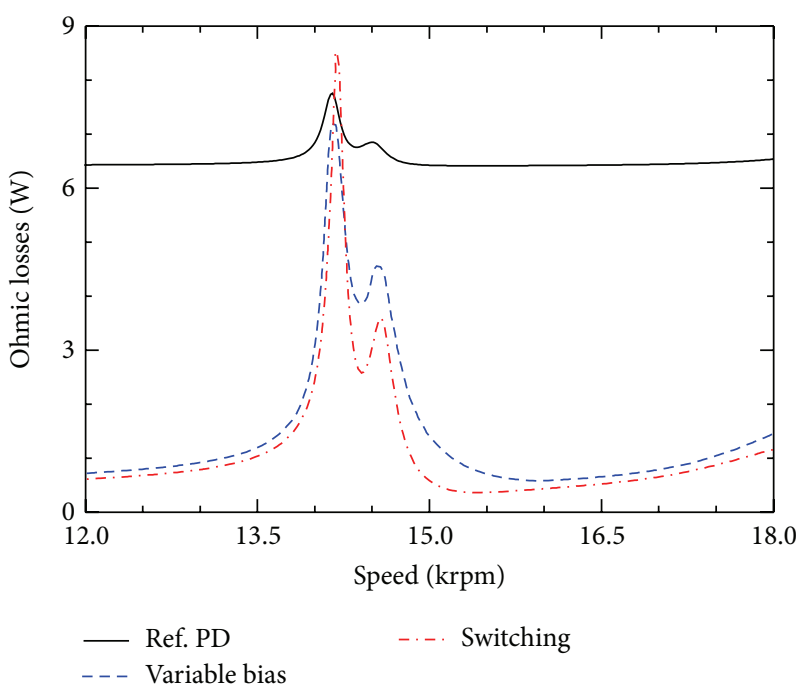

(b)

Figure 11: Unbalance response (ohmic losses).

much smaller currents than the reference controller, at low speeds.

Since it takes less currents for obtaining similar responses when either switching or variable bias control laws are used at low speeds, it is expected that the ohmic losses would be much smaller than what the reference controller produces. Figure 11 shows the total ohmic losses of all radial bearing coils. At low speeds, the power-savings by using either variable bias or switching control laws are almost $90 \%$, when compared to the ohmic losses by the reference controller. However, when the system is operating near the flexible mode, the power consumption by the reference controller is on a par with the power-minimizing laws. In fact, the switching law requires more power to run through the flexible mode, another indication that the controller is not able to stabilize the system.

\section{Conclusions}

Based on the results obtained in this paper, we can conclude that the power-minimizing control laws indeed save a great deal of power, compared to the lead-lag type controller that provides similar closed-loop stiffness. However, the leadlag type controller is more robust than power-minimizing controllers in a sense that it can run through flexible modes while satisfying the requirements without the singularity problem of the switching controller or the adaptation issue with the variable bias controller. 


\section{Conflict of Interests}

The authors declare that there is no conflict of interests regarding the publication of this paper.

\section{Acknowledgment}

This work is supported by a Grant from Korea National Research Foundation (NRF-2009-0075920).

\section{References}

[1] G. Schweitzer and E. H. Maslen, Eds., Magnetic Bearings: Theory, Design, and Application to Rotating Machinery, Springer, New York, NY, USA, 2009.

[2] A. Charara, J. de Miras, and B. Caron, "Nonlinear control of a magnetic levitation system without premagnetization," IEEE Transactions on Control Systems Technology, vol. 4, no. 5, pp. 513-523, 1996.

[3] J. Lévine, J. Lottin, and J.-C. Ponsart, "A nonlinear approach to the control of magnetic bearings," IEEE Transactions on Control Systems Technology, vol. 4, no. 5, pp. 524-544, 1996.

[4] S. Sivrioglu, K. Nonami, and M. Saigo, "Low power consumption nonlinear control with $H_{\infty}$ compensator for a zero-bias flywheel AMB system," Journal of Vibration and Control, vol. 10, no. 8, pp. 1151-1166, 2004.

[5] M. S. de Queiroz and D. M. Dawson, "Nonlinear control of active magnetic bearings: a backstepping approach," IEEE Transactions on Control Systems Technology, vol. 4, no. 5, pp. 545-552, 1996.

[6] P. Tsiotras and B. C. Wilson, "Zero- and low-bias control designs for active magnetic bearings," IEEE Transactions on Control Systems Technology, vol. 11, no. 6, pp. 889-904, 2003.

[7] T. Hu, Z. Lin, and P. E. Allaire, "Reducing power loss in magnetic bearings by optimizing current allocation," IEEE Transactions on Magnetics, vol. 40, no. 3, pp. 1625-1635, 2004.

[8] M. N. Sahinkaya and A. E. Hartavi, "Variable bias current in magnetic bearings for energy optimization," IEEE Transactions on Magnetics, vol. 43, no. 3, pp. 1052-1060, 2007.

[9] D. L. Trumper, S. M. Olson, and P. K. Subrahmanyan, "Linearizing control of magnetic suspension systems," IEEE Transactions on Control Systems Technology, vol. 5, no. 4, pp. 427-438, 1997.

[10] L. Li, "Linearizing magnetic bearing actuators by constant current sum, constant voltage sum, and constant flux sum," IEEE Transactions on Magnetics, vol. 35, no. 1, pp. 528-535, 1999.

[11] ISO, "Mechanical vibration-vibration of rotating machinery equipped with active magnetic bearings-part 1: vocabulary," ISO 14839-1, International Organization for Standardization ISO, 2002.

[12] C. H. Cloud, G. Li, E. H. Maslen, and L. E. Barret, "Practical applications of singular value decomposition in rotordynamics," Australian Journal of Mechanical Engineering, vol. 2, pp. 21-32, 2005.

[13] F. Matsumura and T. Yoshimoto, "System modeling and control design of a horizontal-shaft magnetic-bearing system," IEEE Transactions on Magnetics, vol. 22, no. 3, pp. 196-203, 1986.

[14] H. D. Nelson and J. M. McVaugh, "The dynamics of rotorbearing systems using finite elements," Journal of Engineering for Industry, vol. 98, no. 2, pp. 593-600, 1976.

[15] D. Childs, Turbomachinery Rotordynamics: Phenomena, Modeling, and Analysis, John Wiley \& Sons, New York, NY, USA, 1993.
[16] M. Ito, H. Fujiwara, N. Takahashi, and O. Matsushita, "Evaluation of stability margin of active magnetic bearing control system combined with several filters," in Proceedings of the 9th International Symposium on Magnetic Bearings (ISMB '04), Lexington Ky, USA, 2004.

[17] S. Lei and A. Palazzolo, "Control of flexible rotor systems with active magnetic bearings," Journal of Sound and Vibration, vol. 314, no. 1-2, pp. 19-38, 2008.

[18] R. Herzog, P. Bühler, C. Gähler, and R. Larsonneur, "Unbalance compensation using generalized notch filters in the multivariable feedback of magnetic bearings," IEEE Transactions on Control Systems Technology, vol. 4, no. 5, pp. 580-586, 1996.

[19] ISO 14839-3, "Mechanical vibration-vibration of rotating machinery equipped with active magnetic bearings-part 3: evaluation of stability margin," International Organization for Standardization ISO, 2006. 

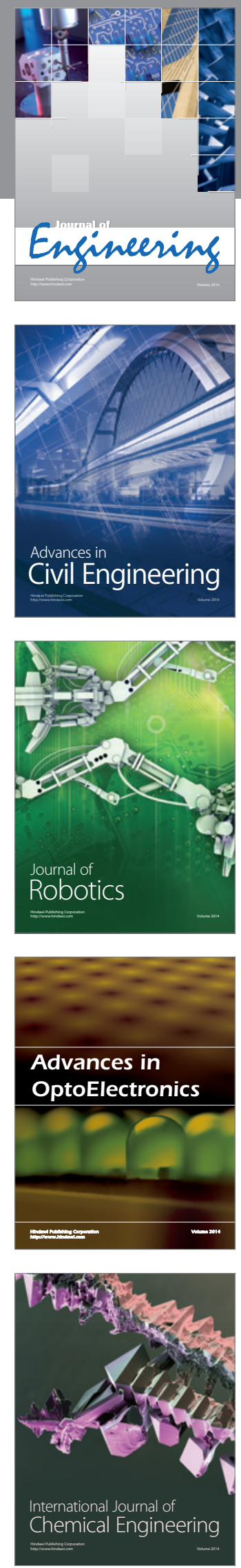

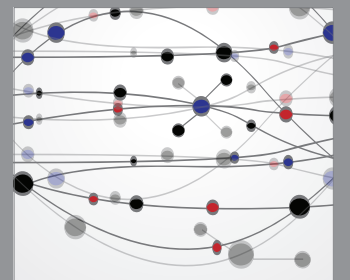

The Scientific World Journal
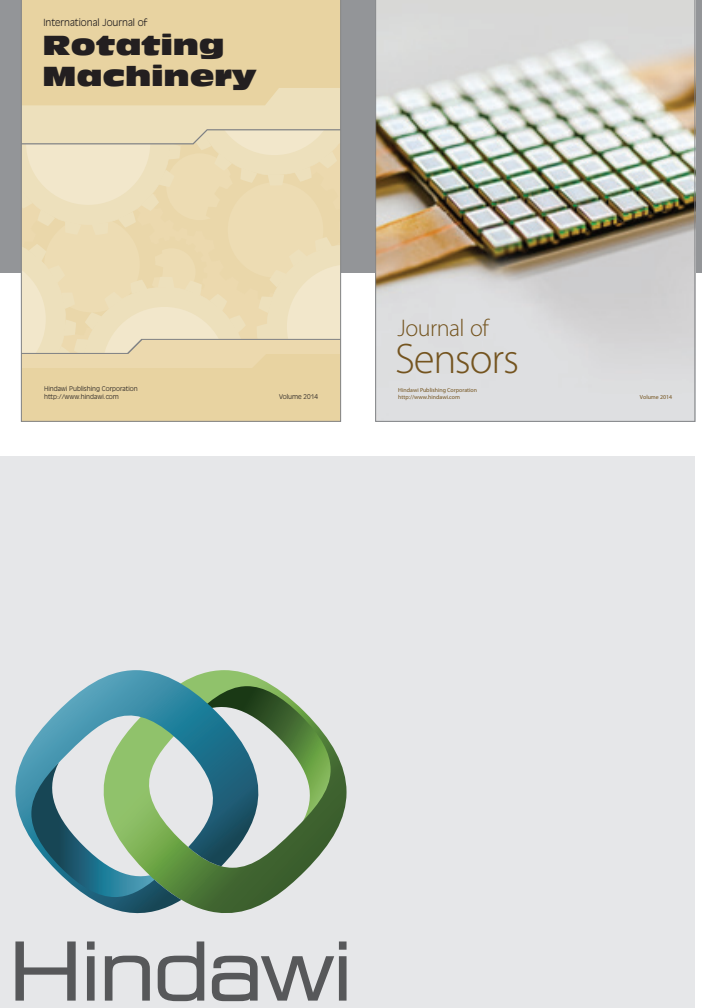

Submit your manuscripts at http://www.hindawi.com
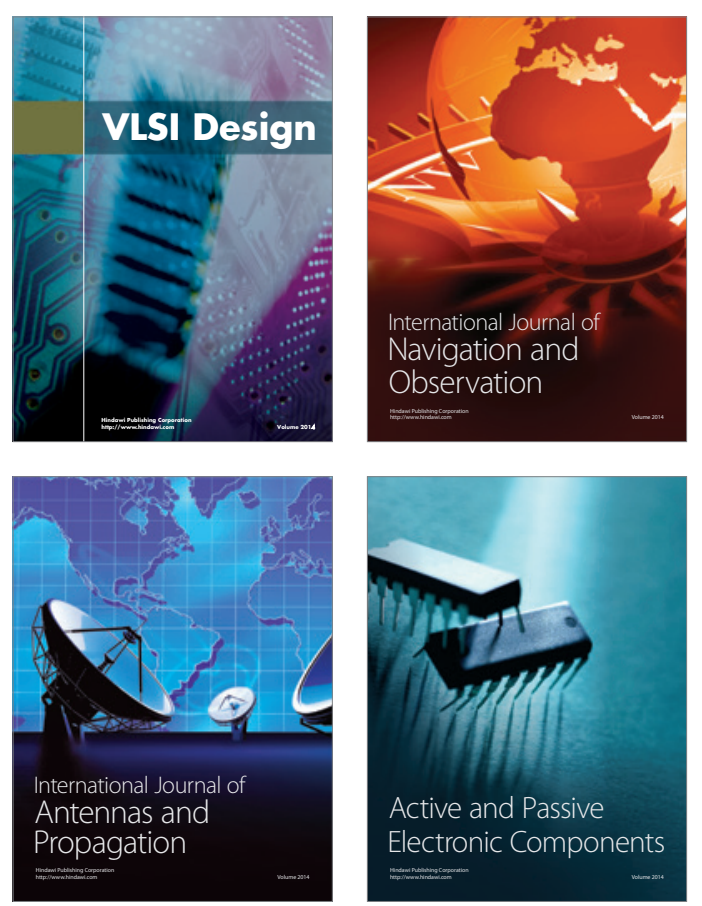
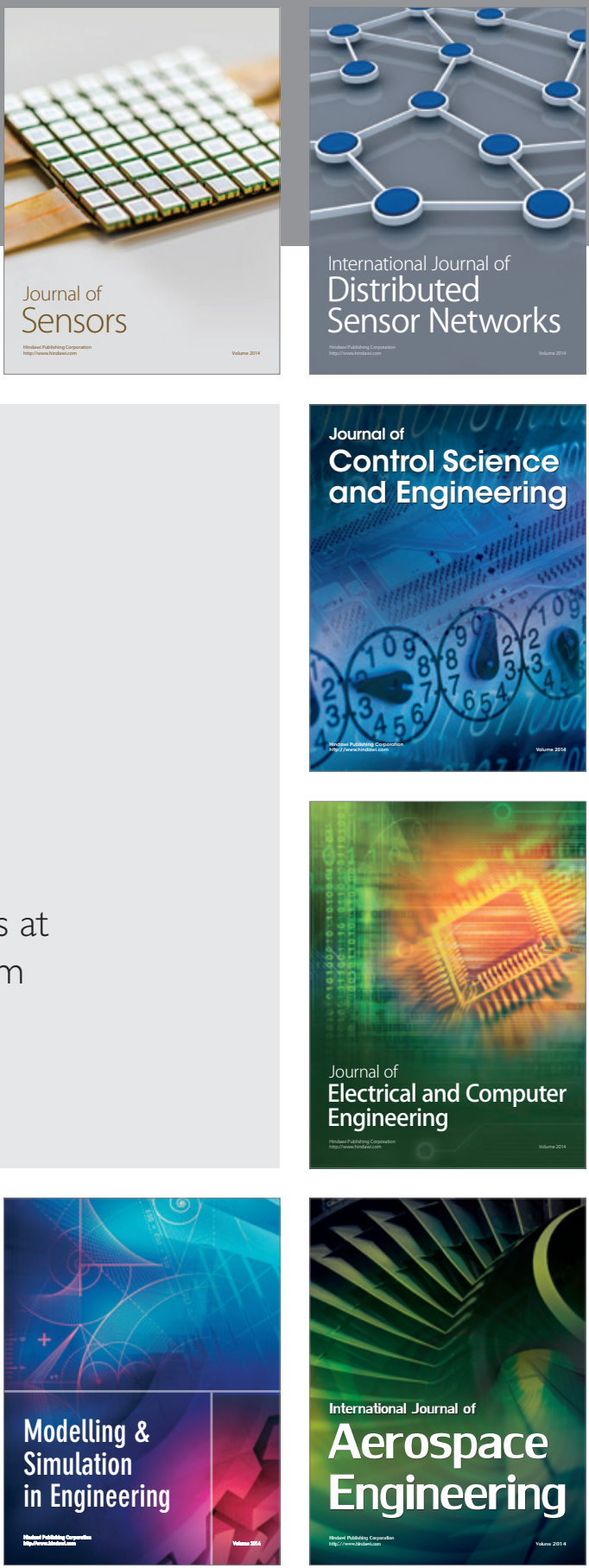

Journal of

Control Science

and Engineering
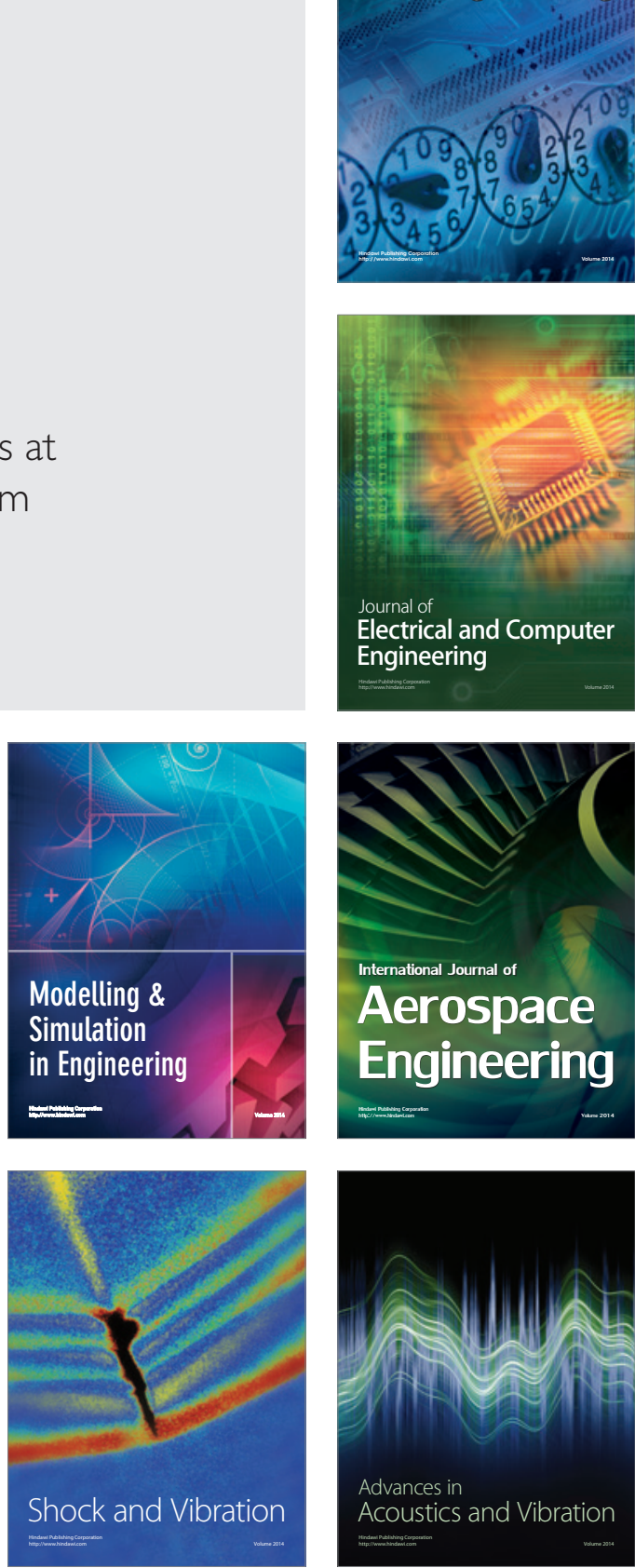